\title{
Optimization of Whole-Body CT Examinations of Polytrauma Patients in Comparison with the Current Diagnostic Reference Levels
}

\section{Optimierung von Ganzkörper-CT-Untersuchungen an Polytraumatisierten anhand des Vergleichs mit den aktuellen diagnostischen Referenzwerten}

Authors

Stefan B. Schäfer ${ }^{1}$, Claudia Rudolph ${ }^{2}$, Martin Kolodziej ${ }^{3}$, Frank Mauermann ${ }^{4}$, Fritz Christian Roller ${ }^{1}$, Gabriele Anja Krombach ${ }^{1}$

\section{Affiliations}

1 Department of Diagnostic and Interventional Radiology, University-Hospital Giessen, Justus-Liebig-University Giessen, Germany

2 Department of Pediatric Radiology, University-Hospital Giessen, Justus-Liebig-University Giessen, Germany

3 Product Management, Infinitt Europe GmbH, Frankfurt, Germany

4 Clinical Application Specialist, Siemens Healthcare GmbH, Erlangen, Germany

Key words

reference levels, dose optimization, whole-body CT, reference $\mathrm{mAs}$

received 13.11.2017

accepted 22.02.2019

Bibliography

DOI https://doi.org/10.1055/a-0881-3113

Published online: 18.4.2019

Fortschr Röntgenstr 2019; 191: 1015-1025

(c) Georg Thieme Verlag KG, Stuttgart · New York ISSN 1438-9029

Correspondence

Stefan B. Schäfer

Department of Diagnostic and Interventional Radiology,

University-Hospital Giessen, Justus-Liebig-University Giessen,

Klinikstraße 33, 35592 Giessen, Germany

Tel.: ++ 49/6 41/98559531

Fax: ++49/641/98541809

stefan.b.schaefer@radiol.med.uni-giessen.de

\section{ABSTRACT}

Purpose Evaluation of the dose values of a polytrauma whole-body CT examination used in clinical practice with regard to the 2016 updated diagnostic reference levels and reduction of the mean exposure levels using simple optimization steps.
Materials and Methods In each case, 100 exposure values before and after dose optimization were compared with the old and new diagnostic reference levels. The grayscale values and the signal-to-noise ratio (SNR) were determined for the lung, the aortic arch and the liver. A visual assessment of the image quality was performed by two radiologists on the basis of a Likert scale ( 0 - non-diagnostic, 1 - poor visualization, 2 moderate visualization, 3 - good visualization, 4 - excellent visualization) for CT examinations both before and after optimization.

Results The acquired exposure values after dose optimization were below the old and new diagnostic reference levels $(1319.98 \pm 463.16 \mathrm{mGy} \cdot \mathrm{cm})$ while the mean value of the exposure values before optimization $(1774.96 \pm 608.78 \mathrm{mGy} \cdot \mathrm{cm})$ exceeded the current diagnostic reference levels. The measured grayscale values $(\mathrm{HU})$ were (before versus after optimization): lung $-833 \mathrm{HU}$ vs. $-827 \mathrm{HU}(\mathrm{p}=0.43)$, aortic arch $341 \mathrm{HU}$ vs. $343 \mathrm{HU}(p=0.70)$ and liver $68 \mathrm{HU}$ vs. $67 \mathrm{HU}(p=0.35)$. After dose optimization the SNR in the lung was minimally higher, while it was minimally lower in the two other regions than before the optimization. Visual assessment of the image quality showed almost identical values with 3.85 evaluation points before and 3.82 evaluation points after dose optimization $(p=0.57)$.

Conclusion Due to the updating of the diagnostic reference levels, an analysis of the own exposure values is necessary in order to be able to detect high values promptly and to initiate appropriate measures for dose reduction. Appropriate adaptation of the examination parameters with consideration of the necessary image quality allows a significant reduction of the radiation exposure in most cases, also on CT devices of older generations.

Key Points:

- In many cases a dose reduction below the DRLs is already possible by optimizing the examination technique.

- In order to ensure a diagnostic image quality, the control of the image quality is unavoidable in a dose reduction.

- Through suitable parameter adjustments a compliance with the DRLs is also possible, using CT devices of older generation without iterative image reconstruction. 


\section{Citation Format}

- Schäfer SB, Rudolph C, Kolodziej M et al. Optimization of Whole-Body CT Examinations of Polytrauma Patients in Comparison with the Current Diagnostic Reference Levels. Fortschr Röntgenstr 2019; 191: 1015-1025

\section{ZUSAMMENFASSUNG}

Ziel Die Überprüfung der Dosiswerte an einem in der klinischen Routine betriebenen Polytrauma-Ganzkörper-CT hinsichtlich der 2016 aktualisierten diagnostischen Referenzwerte (DRW) und Reduktion der mittleren Expositionswerte durch einfache Optimierungsschritte.

Material und Methoden Jeweils 100 Expositionswerte vor und nach der Dosisoptimierung wurden mit den alten und den neuen DRW verglichen. Die Graustufenwerte und das SignalRausch-Verhältnis (SNR) wurden für die Lunge, den Aortenbogen und die Leber bestimmt. Durch 2 Radiologen erfolgte eine visuelle Beurteilung der Bildqualität anhand einer Likert-Skala (0 - nicht diagnostisch, 1 - schlecht sichtbar, 2 - moderat sichtbar, 3 - gut sichtbar, 4 - exzellent sichtbar) sowohl für die CT-Untersuchungen vor als auch nach der Optimierung.

Ergebnisse Die ermittelten Expositionswerte nach der Dosisoptimierung lagen unterhalb der alten und neuen DRW
$(1319,98 \pm 463,16 \mathrm{mGy} \cdot \mathrm{cm})$, während der Mittelwert der Expositionswerte vor der Optimierung $(1774,96 \pm 608,78 \mathrm{mGy} \cdot \mathrm{cm})$ die aktuellen DRW überschritt. Die gemessenen Graustufenwerte $(\mathrm{HE})$ betrugen (vor gg. nach der Optimierung) Lunge $833 \mathrm{HE}$ gg. $-827 \mathrm{HE}(p=0,43)$, Aortenbogen $341 \mathrm{HE} \mathrm{gg.} 343 \mathrm{HE}$ $(p=0,70)$ und Leber $68 \mathrm{HE}$ gg. $67 \mathrm{HE}(p=0,35)$ (gg= gegenüber). Das SNR war nach der Dosisoptimierung in der Lunge minimal höher, in den beiden anderen Regionen minimal geringer als vor der Optimierung. Die visuelle Beurteilung der Bildqualität wies mit 3,85 Bewertungspunkten vor und 3,82 Bewertungspunkten nach der Dosisoptimierung $(p=0,57)$ relativ identische Werte auf.

Schlussfolgerung Anlässlich der Aktualisierung der DRW ist eine Analyse der eigenen Expositionswerte unumgänglich, um Überschreitungen zeitnah erkennen und geeignete Maßnahmen zur Dosisreduktion einleiten zu können. Bereits durch geeignete Anpassung der Untersuchungsparameter unter Berücksichtigung der erforderlichen Bildqualität ist in den meisten Fällen eine deutliche Reduktion der Strahlenexposition, auch bei CT-Geräten der älteren Generationen, möglich.

\section{Introduction}

Due to ongoing technical advances and the further development of established methods in radiology, there is enormous potential for reducing radiation exposure.

Since the X-ray Ordinance came into effect on 9/1/1973 as a federal ordinance, it has regulated radiation protection for patients and examiners. For a long time, exposure for diagnostic purposes was regulated simply by the ALARA principle (as low as reasonably achievable) and there were no concrete exposure restrictions for radiology examinations. The introduction and application of dose reference values were recommended for the first time in 1996 by the International Commission on Radiological Protection (ICRP) [1]. One year later the European Atomic Energy Community (EURATOM) defined diagnostic reference levels (DRLs) as a measure for optimizing radiation protection in “patient protection guidelines" [directive 97/43/ EURATOM] and then confirmed them in the new guidelines 2013/59/EURATOM [2]. Directive 97/43/ EURATOM requires all EU member states to include diagnostic reference levels in their national radiation protection laws and to ensure that these values are regularly reviewed. In radiography and interventional radiology, DRLs are defined as dose values in diagnostic and interventional applications for typical examinations in patients with standard dimensions or in standard phantoms using generally defined equipment. In 1999, the European Commission published guidelines with precise recommendations for introducing DRLs defining the weight of the "standard patient" as $70 \pm 3 \mathrm{~kg}$. In these guidelines, the European Commission also recommends using a mean dose value of at least ten unselected patients separately for every
$X$-ray device in place of the standard patient dose and comparing these values to the relevant DRLs [3].

With inclusion in the amendment to the $\mathrm{X}$-ray Ordinance dated $6 / 18 / 2002$, the DRLs became established for the first time in German (national) law and they must be used as a basis in diagnostic examinations in individuals ( $\$ 16$ paragraph 1 sentence 3 of the $X$-ray Ordinance) [4]. According to $\S 16$ paragraph 1 sentence 3 of the X-ray Ordinance, the Federal Agency for Radiation Protection is responsible for creating and publishing reference values This was performed for the first time on 8/5/2003 in Federal Register No. 143 [4]. The medical authorities are responsible for ensuring compliance with the DRLs for patient radiation exposure [5]. According to $\S 17$ a paragraph 1 sentence 3 no. 2 of the X-ray Ordinance, "constant, unjustified exceeding of these values" must be reported to the responsible regional authorities so that an onsite inspection can be conducted and recommendations for reducing radiation exposure can be made in cooperation with the medical authorities. The Federal Agency for Radiation Protection is responsible for updating the DRLs at regular intervals based on the exposure values of the equipment operators reported annually by the medical authorities. The latest publication of the updated DRLs for diagnostic and interventional X-ray procedures appeared on June 22, 2016 and these values were reviewed at the start of 2017 by the medical authorities [6]. The previous update of the DRLs was published on June 22, 2010 by the Federal Agency for Radiation Protection [7]. The DRLs are based on the 75th percentiles of the distribution of the mean reported patient exposure values and do not represent limit values for individual procedures involving radiation or for patients. The corresponding DRLS must not be exceeded by the mean patient exposure value 
of at least 10 unselected patients for the particular examination type on a unit. The DRLs are specified as the volume CT dose index $\left(C T D I_{V o l}\right)$ and dose length product (DLP) for computed tomography and as the dose area product (DAP) for radiography and interventional radiology. The DRLs published for the first time by the Federal Agency for Radiation Protection on June 22, 2010 were already significantly less than the previously valid reference values for most examinations [7, 8]. The current DRLs are even lower and are also significantly more differentiated.

The equipment operator is responsible for checking whether the own DAP, CTDI ${ }_{\text {Vol }}$ and DLP values satisfy the new requirements and for initiating suitable measures if necessary so that any necessary corrective measures can be implemented to ensure compliance with the new DRLs. Moreover, the lowest possible radiation exposure must be ensured as a function of the state of the art and under consideration of all conditions of the individual case. In addition, it must be assumed that the radiation exposure of the German population as a result of radiology examinations can be further reduced since both the patient dose and thus also the DRLs will be lowered in future updates in the long term and under consideration of technical developments.

The goal of this study was to review the current dose values on the basis of a polytrauma whole-body CT unit operated in the clinical routine in the emergency room of a university radiology institute (Diagnostic and Interventional Radiology) in light of the new DRLs, to identify possibilities for optimization, and to additionally reduce the mean exposure values by means of simple optimization steps. In particular, polytrauma whole-body CT examinations were used for this study because fast and precise clinical and diagnostic imaging methods are essential for patient care. Comparable "whole-body" screening tends to be performed in the case of malignancies in patients with advanced age or possibly with poor prognoses. However, polytrauma patients are often young, e. g. cyclists and motorcyclists involved in an accident, and people with sports injuries or work injuries (e. g. fall from a great height $\geq 3$ meters). Therefore, diagnostic image quality should be achieved with the lowest possible radiation exposure due to the possible biological damage to tissue (e.g. malignant diseases) induced by ionizing radiation which usually only occurs several years after radiation exposure.

\section{Materials and Methods}

The exposure values for anonymized examinations performed on a polytrauma whole-body CT unit (SOMATOM Sensation Open (2011), Siemens, Erlangen, Germany) with the software (Somaris/5 syngo CT 2014A) for the months prior to publication of the new DRLs were taken from the Picture Archiving and Communication System (PACS) and the radiology information system (RIS) for this study. Based on these exposure values, the mean values for the DLP and the CTDI ${ }_{\text {Vol }}$ were calculated and compared to the old and new DRLs.

The CT unit used here is a 24-row unit, the nominal single collimation is $1.2 \mathrm{~mm}$ and the nominal total collimation is $28.8 \mathrm{~mm}$. A voltage of $120 \mathrm{kV}$ and a pitch of 0.65 are used. A body protocol is used for the polytrauma whole-body CT examinations, i.e., a 32-cm test phantom ("body phantom"), and a form filter is used for the trunk. Tube current modulation is performed during the $\mathrm{CT}$ examination in an angle-dependent manner using a biplanar modulation technique. The polytrauma whole-body CT examinations are routine examinations performed approximately 400 times per year. The applied protocol was created in collaboration with an application specialist of the manufacturer.

The images were acquired in a standardized manner using the spiral technique from the base of the skull to the symphysis. A contrast agent (Ultravist ${ }^{\circledR} 370$, Bayer Vital, Leverkusen, Germany) was injected intravenously following a biphasic injection protocol [9]. After the first administration of $50 \mathrm{ml}$ of contrast agent at an injection rate of $3.5 \mathrm{ml} / \mathrm{s}$, the second bolus of $80 \mathrm{ml}$ of contrast agent was injected after a delay of $30 \mathrm{~s}$ with an injection rate of $4.5 \mathrm{ml} / \mathrm{s}$ followed by $50 \mathrm{ml}$ of $\mathrm{NaCl}$ also with an injection rate of $4.5 \mathrm{ml} / \mathrm{s}$. 60 seconds after the first application of contrast agent, the spiral technique begins. Axial reconstruction is performed using the soft tissue window technique with a slice thickness of $5 \mathrm{~mm}$ and using the bone window technique with a slice thickness of $3 \mathrm{~mm}$. An additional reconstruction using the lung window technique was performed for the thorax with a slice thickness of $5 \mathrm{~mm}$. Multiplanar reconstructions are generated using the soft tissue window technique in sagittal and coronal orientation with a slice thickness of $5 \mathrm{~mm}$ and using the bone window technique in sagittal orientation with a slice thickness of $3 \mathrm{~mm}$.

The CT unit being used does not allow iterative image reconstruction which can be used to reduce the necessary radiation exposure while maintaining image quality using computationally intensive image reconstruction algorithms. With iterative image reconstruction, a dose reduction of up to approx. $75 \%$ can be achieved [10]. However, in the case of a significant dose reduction (> $40 \%$ ), there is a risk that lesions with low contrast enhancement can no longer be detected [11]. Therefore, other suitable optimization options had to be sought following the realization that the calculated mean dose values were in compliance with the old reference values but were higher than the new DRLs. Possibilities for reducing radiation exposure while maintaining diagnostic image quality by simply changing $\mathrm{CT}$ imaging parameters were developed and implemented in collaboration between physicians and physicists. The slice-dependent mAs reference value (referred to as the "mAs reference value" in the following) was reduced from $165 \mathrm{mAs}$ to $130 \mathrm{mAs}$. This reference value determines image quality and can be accessed by users, i. e., they can perform the change and are not reliant on the manufacturer. The mAs reference value is specified per rotation and on this CT unit is based on a $75-80 \mathrm{~kg}$ patient (standard patient as defined by the manufacturer). Based on this mAs reference value, the tube current modulation is performed as a function of the patient attenuation (patient diameter). In the case of the unit used here, this is achieved in an angle-dependent manner by a biplanar (double) tube current modulation on the one hand in the z-direction based on the topogram using the patient-specific attenuation profile and on the other hand in the $\mathrm{x}$ - and $\mathrm{y}$-directions (lateral and posterior) during the scan. This means that the dose arriving at the detector is detected and the effective mAs value listed in the patient protocol is regulated up or down based on the " $\mathrm{mAs}$ refer- 
ence vale". Reduction of the mAs reference value results in a reduction in dose but at the same time in an increase in image noise.

Mean values consisting of 100 examinations before and after optimization of the system were used for the evaluation. The calculated values were compared with the old and the new DRLs and were analyzed. A DLP of $1798 \mathrm{mGy} \cdot \mathrm{cm}$ according to the old DRLs and a DLP of $1330 \mathrm{mGy} \cdot \mathrm{cm}$ according to the new DRLs were assumed for the examination on the polytrauma whole-body CT unit from the base of the skull to the symphysis. For this purpose, the specified reference DLP values for the facial bones (sinusitis), thorax, abdomen, and pelvis according to the old DRLs were added. Since the specified DRLs for the facial bones are based on the 16-cm test phantom ("head phantom"), this value could not be easily added to the remaining DRLs that are based on the $32-\mathrm{cm}$ test phantom. This value was converted using the phantom factors of the unit so that it was decreased from $100 \mathrm{mGy} \cdot \mathrm{cm}$ to $48 \mathrm{mGy} \cdot \mathrm{cm}$. The DLP reference value for the torso examination region (thorax + abdomen + pelvis) was added to the DLP reference value for the neck examination region according to the new DRLs. Since the specified reference values for the two added examination regions are based on the "body phantom", the DLPs can be easily added. However, addition of the individual reference values for the various examination regions according to both the old and the new DRLs results in overlapping of the reference values according to the data in the guidelines of the German Medical Association [12].

The size and weight of the patients was taken from the hospital information system (HIS) and the body mass index (BMI) of the patients was determined on the basis of this data. The patients were then categorized according to the individual degrees of obesity as defined by the WHO [13] so that this information could be used for evaluation if patients greatly exceeded and negatively influenced the mean values for DLP and CTDI ${ }_{\text {Vol }}$ due to a high BMI.

In the case of patients without corresponding data in the HIS, conclusions about their BMI were made based on visual impressions and examination of the subcutaneous fat tissue on the CT images. Since this was performed without technical tools based on the CT images, uncertainty regarding the estimated BMI cannot be completely ruled out.

The various subgroups of degrees of obesity according to the WHO definition with a patient number $>10$ both before and after optimization were examined separately in greater detail. In particular, attention was paid to the presence of metallic implants.

For objective assessment of image quality, the grayscale values representing the attenuation of X-rays in tissue (Hounsfield units, $\mathrm{HU}$ ) in the lung, liver, and aortic arch were determined for all evaluated examinations. Although $\mathrm{CT}$ units are HU-calibrated, the possibility of significant noise caused by a change was to be ruled out in this study by checking the mean HU values. In addition, the signal-to-noise ratio (SNR) was determined based on the mean signal intensity $\mathrm{P}_{\text {Signal }}$ and the mean noise signal in an area of $10 \times 10$ pixels [14]. Every examination included three regions of interest: ROI 1 in the lung, ROI2 in the aortic arch, and ROI3 in the liver.

Visual image analysis of the examinations was then performed using the Likert scale ( 0 - non-diagnostic, 1 - poor visualization,
2 - moderate visualization, 3 - good visualization, 4 - excellent visualization) [15-17]. Image quality was assessed visually by two radiologists with 21 and 7 years of professional experience. Examinations were evaluated in a randomized and anonymized manner so that it was not clear whether the examinations were performed before or after the parameter change. For all individual assessments by the two observers, the mean value was calculated separately for the CT examinations before and after the parameter change to achieve a better comparison. A two-sided t-Test was then performed both for the objective assessment and for the visual assessment of image quality to check the data for significant differences.

\section{Results}

The dose averaging analysis shows that both the old and the new DRLs are satisfied in the CT examinations performed after the reduction of the mAs reference value from $165 \mathrm{mAs}$ to $130 \mathrm{mAs}$ while the old DRLs are satisfied but the new DRLs are exceeded prior to the parameter change.

- Fig. 1 provides an overview of the mean values for the CT examinations performed on the polytrauma whole-body CT unit in the emergency room with comparison of the old and the new DRLs > Fig. 1.

This graphic shows that the values for the CT examinations performed after the change in mAs reference value was significantly less than the old and slightly less than the new DRLs. Therefore, the DLP values after the parameter change (mean value $1319.98 \pm 463.16 \mathrm{mGy} \cdot \mathrm{cm})$ correspond to approx. $71.4 \%$ of the old DRLs and approx. $99.2 \%$ of the new DRLs. The mean value for the CT examinations performed prior to the parameter change $(1774.96 \pm 608.78 \mathrm{mGy} \cdot \mathrm{cm})$ corresponds to approx. $95.9 \%$ of the old DRL but would exceed the new DRL by approx. $33.5 \%$. The $\mathrm{CTDI}_{\mathrm{vol}}$ values for the examinations performed after optimization are $14.32 \pm 4.48 \mathrm{mGy}$, while the $\mathrm{CTDI}_{\mathrm{vol}}$ before optimization has a mean value of $18.01 \pm 4.54 \mathrm{mGy}$. The distribution of values for $\mathrm{DLP}(\mathrm{A})$ and $\mathrm{CTDI}_{\mathrm{vol}}(\mathrm{B})$ in relation to the $\mathrm{CT}$ examinations before and after lowering of the $m A s$ reference value is shown in a graphic in Fig. 2 . The average scan length was $92.76 \pm 13.06 \mathrm{~cm}$ (minimum: $62.94 \mathrm{~cm}$; maximum $142.94 \mathrm{~cm}$ ) in CT examinations performed after the parameter change and $94.83 \pm 15.44 \mathrm{~cm}$ (minimum: $76.34 \mathrm{~cm}$; maximum $147.44 \mathrm{~cm}$ ) in CT examinations performed before the parameter change.

The t-test for the dose values for the examinations performed prior to and after reduction of the $\mathrm{mAs}$ reference value shows that the radiation exposure could be significantly reduced $(p<0.0000001)$ by the parameter change.

There is a striking difference between the lowest and the highest value of the dataset for both the CT examinations performed before dose optimization and those performed after. Some outliers that greatly exceed the DRL are due to the presence of metallic implants in the patients. However, these extreme values are due in most cases to extremely overweight patients who greatly exceed the standard patient weight of $70 \pm 3 \mathrm{~kg}$ as defined in the EURATOM “patient protection guidelines". 


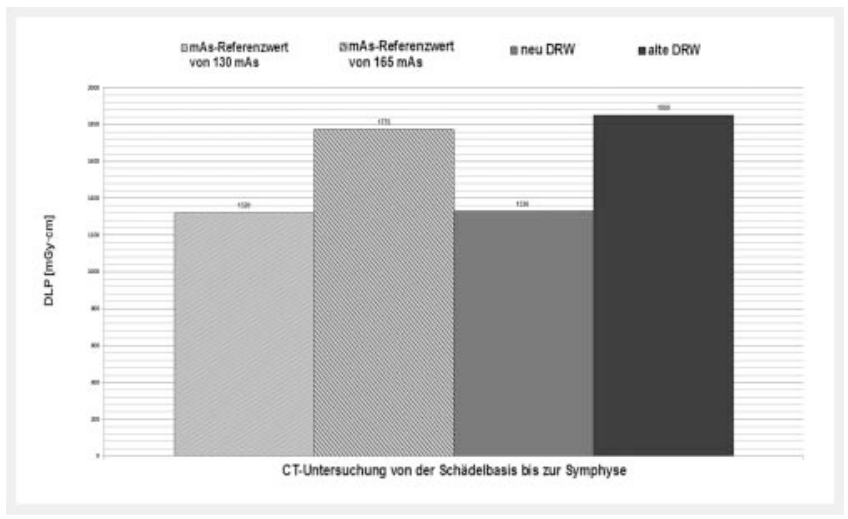

- Fig. 1 Mean DLP values for CT examinations from the base of the skull to the symphysis performed on a polytrauma whole-body CT unit before and after reduction of the "mAs reference value".

- Fig. 3 shows a boxplot of the distribution of patients according to the degrees of obesity as defined by the WHO for CT examinations with an $\mathrm{mAs}$ reference value of 130 and with a value of $165 \mathrm{mAs}$. It shows that the majority of patients were overweight, i. e., over the weight of $70 \pm 3 \mathrm{~kg}$ defined by the European Commission as the "standard patient" in the CT examinations both before and after the parameter change, thus negatively affecting the mean dose value. Approx. $73 \%$ of patients were overweight in the examinations prior to dose optimization, while approx. $65 \%$ of patients were overweight in the CT examinations after reduction of the $m A s$ reference value.

Due to the different distribution of patient BMI in the two groups, the patients were classified in subgroups according to degree of obesity as defined by the WHO and the subgroups with more than 10 patients before and after the reduction of the mAs reference value were examined more closely. This included the following degrees of obesity: "normal weight", "obesity class I", and "obesity class II".

This evaluation within the individual degrees of obesity shows that the mean DLP and CTDI ${ }_{\text {vol }}$ values for the CT examinations performed before and after the optimization steps are significantly lower than the valid DRLs. The values for examinations performed after the parameter change are significantly less than the values for examinations performed prior to the parameter change. Therefore, the mean values for examinations with an $\mathrm{mAs}$ reference value of 130 are $1030.97 \pm 207.00 \mathrm{mGy} \cdot \mathrm{cm}$ for the DLP and $11.44 \pm 2.11 \mathrm{mGy}$ for $\mathrm{CTDI}_{\mathrm{vol}}$ at a scan length of $89.92 \pm 2.79 \mathrm{~cm}$ (minimum: $81.28 \mathrm{~cm}$; maximum $94.36 \mathrm{~cm}$ ). In examinations with an $\mathrm{mAs}$ reference value of $165 \mathrm{mAs}$, the values are $1345.97 \pm$ $217.98 \mathrm{mGy} \cdot \mathrm{cm}$ for the DLP and $14.36 \pm 2.46 \mathrm{mGy}$ for $\mathrm{CTDI}_{\mathrm{vol}}$ at a scan length of $93.62 \pm 2.67 \mathrm{~cm}$ (minimum: $88.79 \mathrm{~cm}$; maximum $98.31 \mathrm{~cm}$ ). A graphic representation of the distribution of the values for $\mathrm{DLP}(\mathrm{A})$ and $\mathrm{CTDI}_{\text {vol }}(\mathrm{B})$ in normal-weight patients is provided in $>$ Fig. 4.

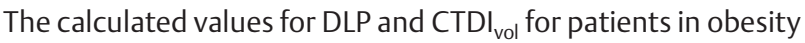
class I are below the relevant DRLs for CT examinations performed both before and after the parameter change. At a scan length of $91.53 \pm 14.14 \mathrm{~cm}$ (minimum: $61.48 \mathrm{~cm}$; maximum $106.42 \mathrm{~cm}$ ), the values for DLP $(1275.07 \pm 267.73 \mathrm{mGy} \cdot \mathrm{cm})$ and for CTDI ${ }_{\mathrm{vol}}$

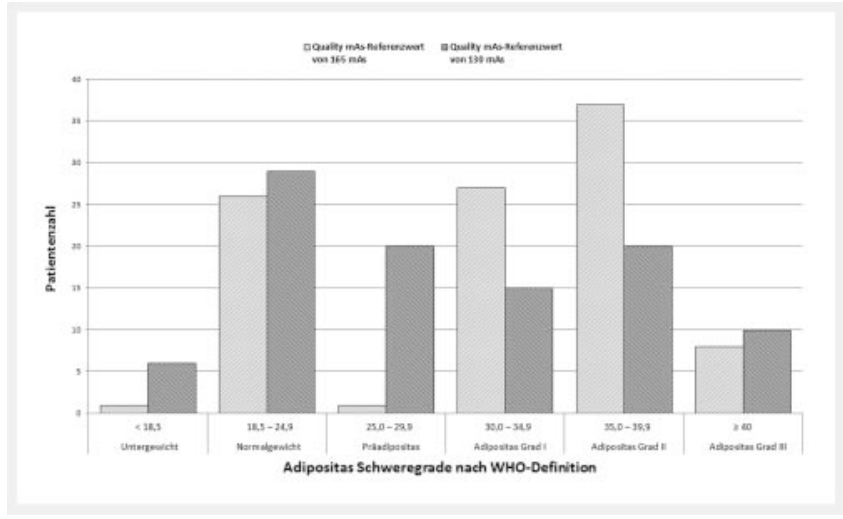

- Fig. 2 Frequency distribution of patients based on degrees of obesity according to the WHO definition. Patients undergoing CT examinations before and after the change of the $\mathrm{mAs}$ reference value.

(14.04 $\pm 2.58 \mathrm{mGy}$ ) for examinations with an $\mathrm{mAs}$ reference value of 130 were significantly lower than the values for examinations with an mAs reference value of 165 (DLP: 1644.28 $398.46 \mathrm{mGy} \cdot \mathrm{cm}, \mathrm{CTDI}_{\mathrm{vol}}: 16.78 \pm 3.01 \mathrm{mGy}$, scan length: $93.68 \pm$ $12.29 \mathrm{~cm}$ (minimum: $81.62 \mathrm{~cm}$; maximum $132.48 \mathrm{~cm}$ )). A detailed representation of the distribution of the values for patients in obesity class I is shown in - Fig. $\mathbf{5}$. The evaluation of the subgroup of patients in obesity class II showed that the values after the optimization steps for DLP $(1573.27 \pm 442.29 \mathrm{mGy} \cdot \mathrm{cm})$ and for $\mathrm{CTDI}_{\text {vol }}(17.63 \pm 3.98 \mathrm{mGy})$ at a scan length of $89.52 \pm$ $16.57 \mathrm{~cm}$ (minimum: $62.94 \mathrm{~cm}$; maximum $142.94 \mathrm{~cm}$ ) were significantly below the values for DLP $(1385.84 \pm 447.38 \mathrm{mGy} \cdot \mathrm{cm})$ and $\mathrm{CTDI}_{\text {vol }}(19.5 \pm 3.77 \mathrm{mGy})$ prior to optimization at an average scan length of $92.98 \pm 14.91 \mathrm{~cm}$ (minimum: $80.32 \mathrm{~cm}$; maximum $131.00 \mathrm{~cm}$ ). Fig. 6 shows a graphic of the distribution of DLP and $\mathrm{CTDI}_{\mathrm{vol}}$ for patients in obesity class II.

The calculated values for the objective assessment of the image quality showed no significant differences between examinations performed before and after the parameter change with respect to grayscale values and signal-to-noise ratio ( $\triangleright$ Table $\mathbf{1}$ ).

The mean grayscale values for CT examinations in the lung were $-833 \mathrm{HU}$ prior to dose optimization and $-827 \mathrm{HU}$ after optimization ( $p=0.43$ ) The mean values for the grayscale values in the aortic arch were $341 \mathrm{HU}$ at an mAs reference value of $165 \mathrm{mAs}$ and $343 \mathrm{HU}(\mathrm{p}=0.70)$ at an $\mathrm{mAs}$ reference value of $130 \mathrm{mAs}$. Only minimal differences regarding the mean values before and after the parameter change (68 HU before and $67 \mathrm{HU}(\mathrm{p}=0.35)$ after reduction of the $\mathrm{mAs}$ reference value) were also seen in the liver. There was also no significant difference between the CT examinations performed before and after dose optimization regarding the signal-to-noise ratio values. In the lung the SNR after the parameter change (mean value 112.08) was slightly better than in the examinations before the parameter change (mean value 108.24) $(p=0.72)$. In the aortic arch and the liver, the SNR in the examinations performed prior to the parameter change was slightly higher than in the subsequent examinations with an $\mathrm{mAs}$ reference value of $130 \mathrm{mAs}$. The SNR value in the aortic arch was 59.27 prior to the reduction of the $\mathrm{mAs}$ reference value and 56.13 


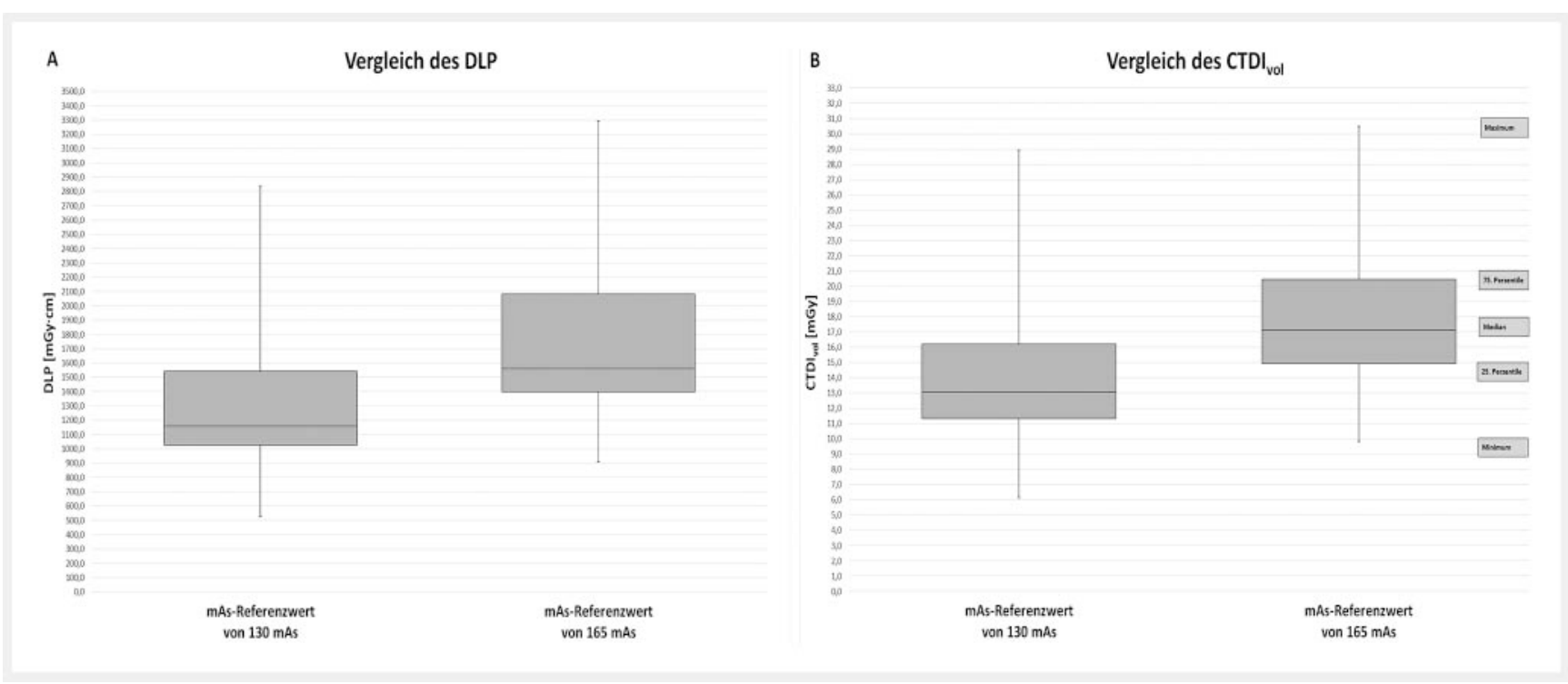

- Fig. 3 Boxplot of the comparison of DLP and CTDlvol for CT examinations before and after the change of the mAs reference value.

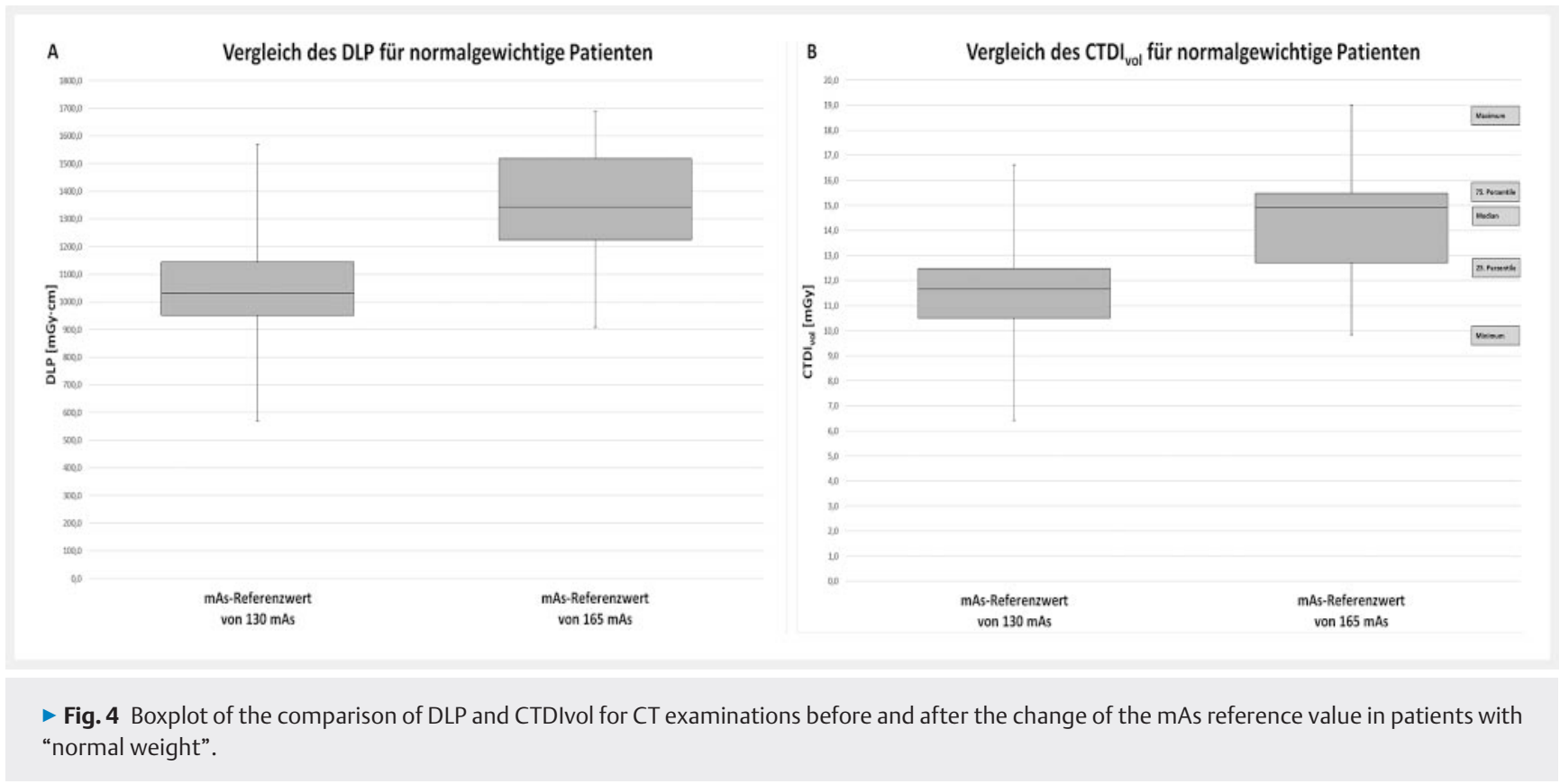

after the change $(p=0.22)$. For the liver the signal-to-noise ratio values were 12.35 prior to the parameter change and 11.07 $(p=0.21)$ after the parameter change.

The visual assessment of image quality by two radiologists using the Likert scale showed relatively identical ratings of image quality before and after dose optimization, thus indicating that the diagnostic image quality was not affected by the adjustment of the mAs reference value ( $\triangleright$ Fig. $7 \mathbf{a}, \mathbf{b})$. The CT scan images with 3.85 evaluation points at an $\mathrm{mAs}$ reference value of $165 \mathrm{mAs}$ and with 3.82 evaluation points $(p=0.57)$ at an $m A$ s reference value of 130 also did not show any significant differences with respect to the ability to assess image quality.

\section{Discussion}

The new DRLs are defined by the Federal Agency for Radiation Protection based on the 75th percentiles of the average dose values reported by equipment operators to the medical authorities and are updated at regular intervals, with the last update having been performed on June 22, 2016. This concept will result in a reduction of patient radiation exposure in the long-term as long as technical developments allow further dose reduction. Approx. $25 \%$ of the dose values reported by equipment operators exceed the new reference values according to the DRL update from June 22, 2016 so that equipment operators must reduce the radiation exposure for every examination type in which the mean dose 


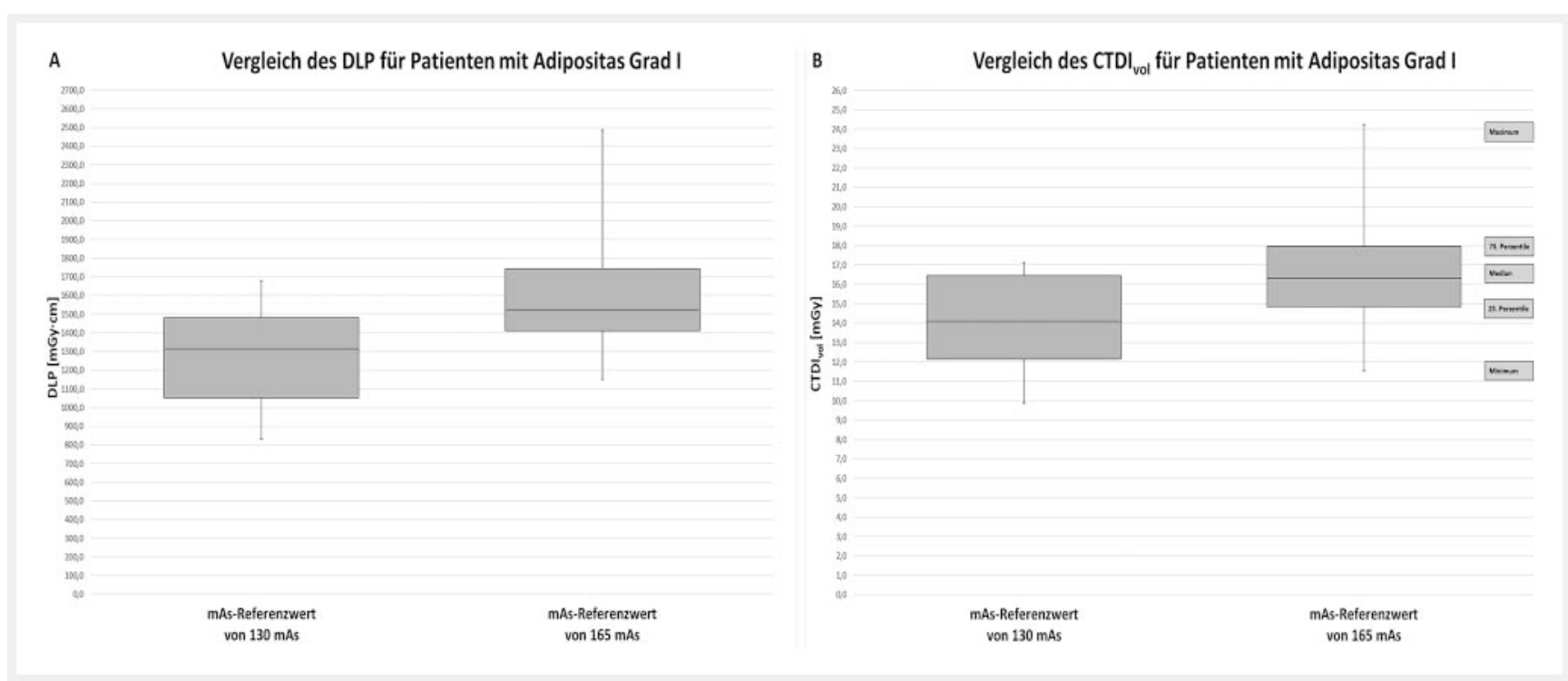

Fig. 5 Boxplot of the comparison of DLP and CTDlvol for CT examinations before and after the change of the mAs reference value in patients in obesity class I.

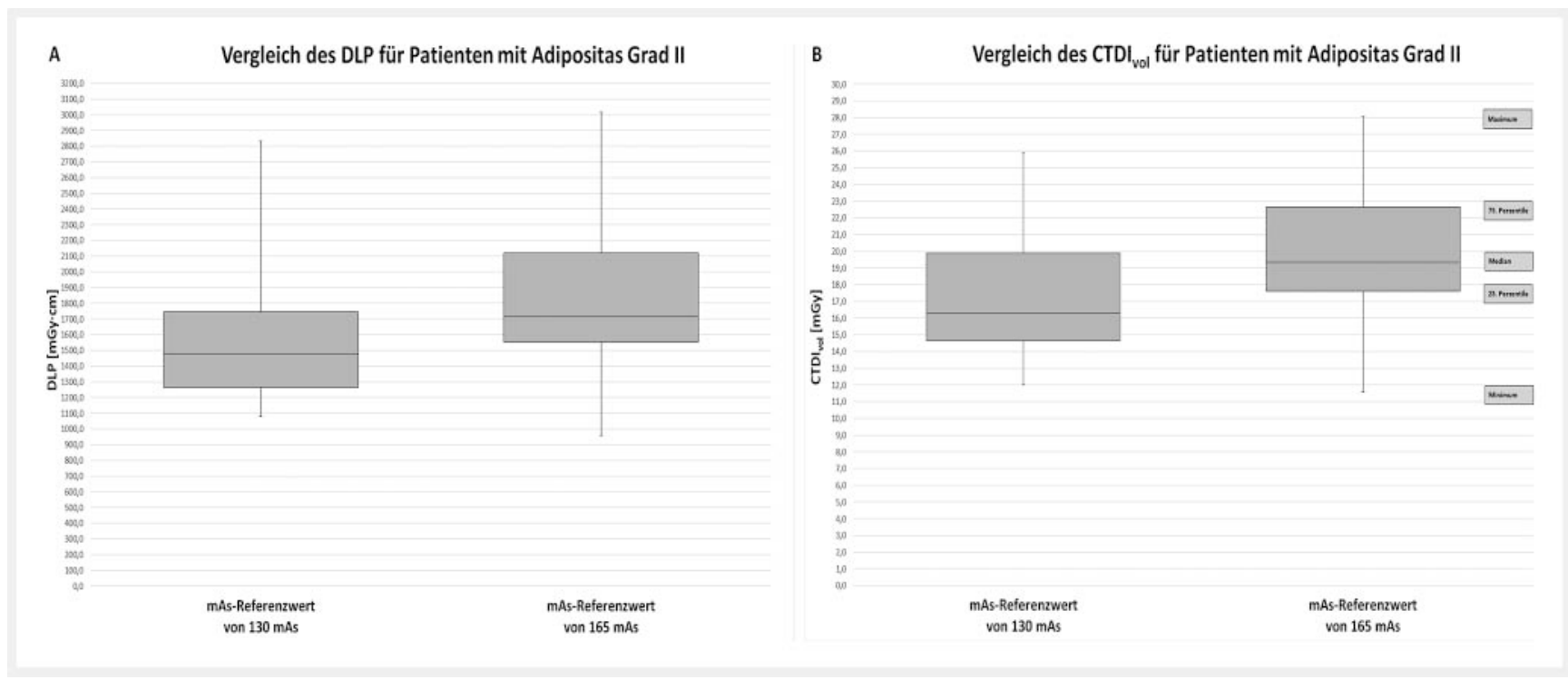

D Fig. 6 Boxplot of the comparison of DLP and CTDIvol for CT examinations before and after the change of the mAs reference value in patients in obesity class II.

values exceed the new DRLs. Optimization of the examination technique by changing the imaging parameters is often already sufficient to lower the radiation exposure enough to satisfy the DRLs. In addition to optimization of the imaging parameters, this use of differently weighted protocols, adapted to the clinical condition of the patient, is an interesting possibility for dose reduction [18]. However, strict monitoring of the image quality is necessary to ensure diagnostic image quality despite the dose reduction. If the mean radiation exposure cannot be reduced to less than the DRLs, service technicians employed by the equipment manufacturer and/or the medical authority need to be involved or in extreme cases an equipment upgrade or even replacement of the equipment is necessary.

This study shows that a significant reduction of radiation exposure is possible even in the case of CT equipment of older generations without iterative image reconstruction in the case of suitable adjustment of the examination parameters under consideration of the required image quality. Although the majority of patients (65-70\%) in the CT examinations evaluated here were overweight and thus exceeded the "standard patient" body weight of $70 \pm 3 \mathrm{~kg}$ defined by the European Commission, the radiation exposure could be reduced by approx. $25.5 \%$ and thus reduced to less than the new DRLs by lowering the mAs reference value. 
- Table 1 Mean values of the gray levels (HU) and SNR for the assessed anatomical structures for CT examinations before and after the reduction of the "mAs reference value", as well as the p-values of the t-Test for significance.

\begin{tabular}{|l|l|l|l|l|l|l|}
\hline & \multicolumn{2}{|l|}{$\mathbf{m A s}$ reference value of $\mathbf{1 6 5} \mathbf{m A s}$} & mAs reference value of $\mathbf{1 3 0}$ mAs & two-sided t-test (p-value) \\
\hline anatomical structure & $\varnothing$ Grayscale values (HU) & $\varnothing$ SNR & $\varnothing$ Grayscale values (HU) & $\varnothing$ SNR & Grayscale values \\
\hline lung & -833 & 108.24 & -827 & 112.08 & 0.43 & SNR \\
\hline aortic arch & 341 & 59.27 & 343 & 56.13 & 0.70 & 0.72 \\
\hline liver & 68 & 12.35 & 67 & 11.07 & 0.35 & 0.22 \\
\hline
\end{tabular}
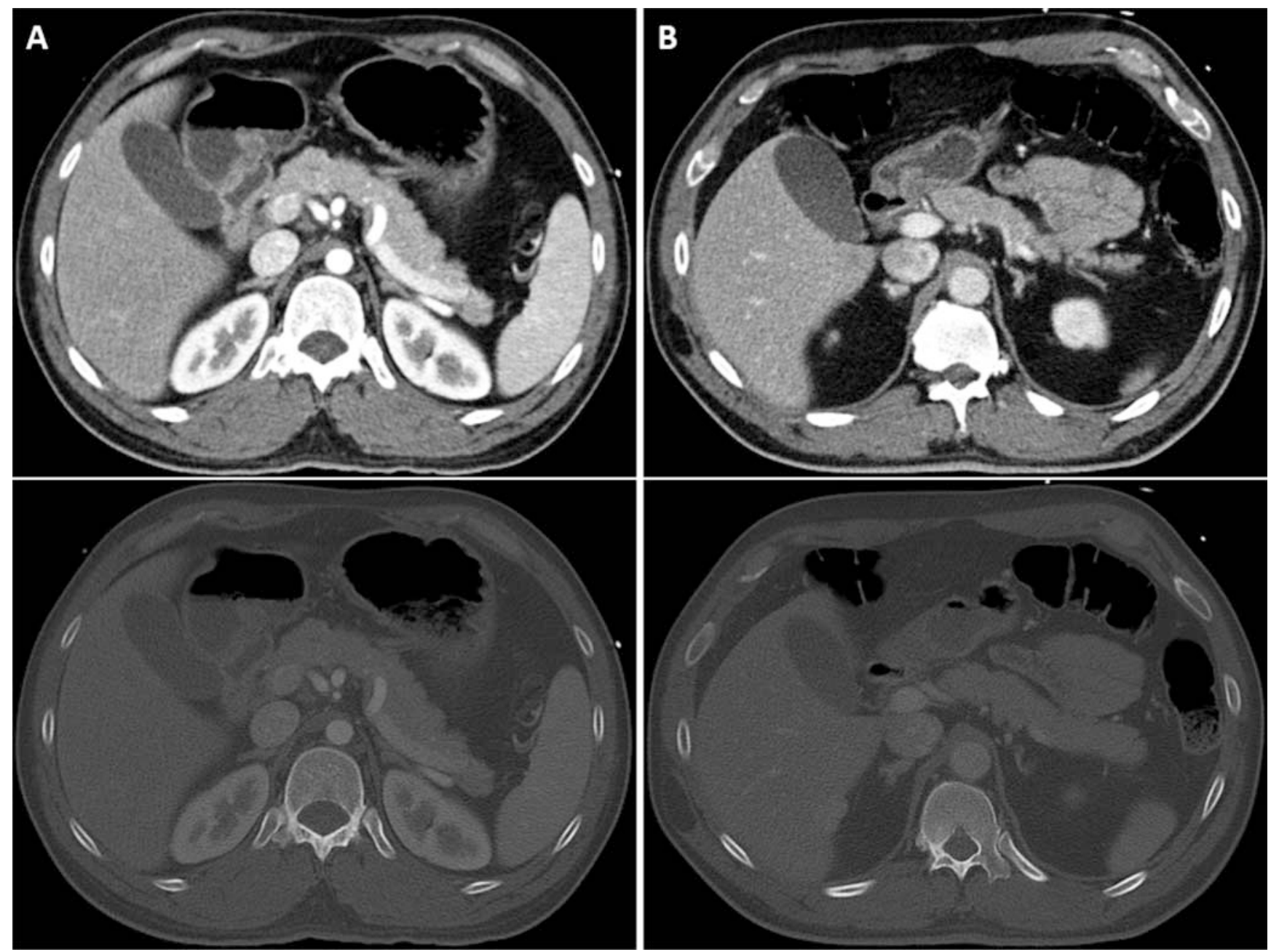

> Fig. 7 CT images before $\mathbf{a}$ and after $\mathbf{b}$ the dose optimization in two different patients using the soft tissue and bone window technique.

However, since the addition of the individual reference values for the various examination regions results in overlapping of the reference values according to the specifications of the guidelines of the German Medical Association, the actual reduction of the radiation exposure is probably slightly smaller.

Therefore, there is an overlap region of a few centimeters between the cervicothoracic junction and the aortic arch for the neck (facial bones to aortic arch) and torso (thorax + abdomen + pelvis) examination regions according to the new DRLs so that it can be assumed that the DRL is slightly lower than the calculated DLP value of $1330 \mathrm{mGy} \cdot \mathrm{cm}$.

For the facial bone (upper edge of the frontal sinus to the chin) and thorax examination regions, there is a region between the chin and cervicothoracic junction that is not taken into consideration by the old DRLs but is scanned in whole-body examinations. However, the thorax and abdomen examination regions (overlap region between the dome of the diaphragm and the dorsal recess of the diaphragm) and abdomen and pelvis examination regions 
(overlap region between the aortic bifurcation and pelvic floor) result in small $(\leq 10 \mathrm{~cm})$ to medium $(\leq 20 \mathrm{~cm})$ overlapping of the reference values according to the specifications of the guidelines of the German Medical Association [12]. As a result of this overlapping of the reference values, the calculated DLP value of 1798 $1798 \mathrm{mGy} \cdot \mathrm{cm}$ for the DRL is slightly high.

The comparison with other current studies on CT units with the option of iterative image reconstruction yielded similar DLP and CTDIvol values [19]. However, the study did not provide any data regarding the weight or $\mathrm{BMI}$ of the included patients so that it is difficult to determine whether only patients with the weight of a "standard patient" or also overweight patients were included in the study. Since the DRLs relate to a "standard patient", evaluating all values by adding the dose values of both normal-weight and overweight patients to calculate an average value usually results in significantly higher exposure values. This is also seen when evaluating the results for DLP and CTDI ${ }_{\text {vol }}$ in the subgroups according to the degrees of obesity defined by the WHO. Due to the different distribution of patient BMI in the two groups, the patients were classified in subgroups according to degree of obesity as defined by the $\mathrm{WHO}$ and the subgroups with more than 10 patients before and after the reduction of the $\mathrm{mAs}$ reference value were examined more closely. This included the following degrees of obesity: "normal weight", "obesity class I", and "obesity class II".

This evaluation within the individual degrees of obesity shows that the mean DLP and CTDI ${ }_{\text {vol }}$ values for the CT examinations performed before and after the optimization steps are significantly lower than the valid DRLs. The average dose values of patients with "normal weight" are significantly below the DRLs, while the dose values for patients in "obesity class I" are barely less than the DRLs. In patients in "obesity class II", the dose values already clearly exceed the DRLs. The values for examinations performed after the parameter change are significantly less than the values for examinations performed prior to the parameter change. Consequently, as a result of the high number of overweight patients in an obesity class of I or higher in this study, the values are only barely less than the new DRLs even after the parameter change. In light of the fact that the BMI of patients without weight and size information in the HIS was determined based on visual impressions and examination of the subcutaneous fat tissue on the CT scan images, slight uncertainty regarding the calculation of the BMI cannot be completely ruled out. Due to the relatively large range of the individual degrees of obesity, this uncertainty is only relevant in the case of patients with a BMI near the limit between two degrees of obesity and this was not seen in the patients in this study. However, limits must also be taken into consideration in calculated BMIs in clinical practice. Since BMI calculation is independent of sex and age and there is no differentiation between lean body mass, muscle mass, and fat mass, there can be limitations regarding the exactness of BMI also in this case [20]. Consequently, the uncertainty in the determination of BMI based on visual impressions and the evaluation of subcutaneous fat tissue is so minimal compared to arithmetically calculated BMI that the exactness of the classification to the individual degrees of obesity was not affected.
For this study, the exposure values of patients who greatly exceeded the weight of the "standard patient" were included in the calculation of the mean dose value, while exceeding of the DRLs in such cases is recorded in the monitoring and evaluation by the medical authority and the Federal Agency for Radiation Protection as justified exceeding of the DRLs. The polytrauma CT unit that was used here is characterized by a particularly large gantry diameter $(82 \mathrm{~cm})$. This design is supposed to simplify positioning of severely injured patients and facilitates access to patients. However, the greater distance between the X-ray tube and the detector results in the need for a higher dose compared to equipment with a smaller diameter in order to generate images of the same quality. The visual assessment of image quality showed comparable image quality without significant differences between the quality of CT scan images acquired before and after the parameter change. This is also the case for signal-to-noise ratio as a parameter for the objective assessment of image quality which has already become established in previous studies in various regions for the evaluation of CT scan images [21, 22]. Based on the results of the visual and objective evaluation of image quality and with respect to the slightly lower values compared to the new DRLs, a further dose reduction based on suitable parameter changes is possible as long as diagnostic image quality according to the ALARA principle can be ensured.

In addition to the possibility to reduce radiation exposure while maintaining diagnostic image quality using simple optimization steps like adjustment of the examination parameters, there is also the possibility of independent dose management that exceeds the legal requirements.

If the mean dose values are less than the current DRLs during the review by the medical authority, the medical authority does not issue a warning even in the event of a continuous increase in exposure values over a longer period of time. Consequently, changes in radiation exposure resulting from small changes in parameters in the course of operation can go unnoticed. By analyzing the own archived exposure values and reviewing and comparing them with the dose applied to date, it is possible to detect dose deviations in a timely fashion, to identify causes, and to implement suitable measures. Thus, radiation exposure can now be controlled on a continuous basis as required by the X-ray Ordinance instead of only on a retrospective basis by the medical authority. However, documentation of the type of examination, the applied dose, the X-ray unit being used, and the weight of the patient being examined is particularly relevant here. This study highlights the potential for operators of $\mathrm{X}$-ray equipment to review their own archived exposure values in order to optimize the applied dose and for the purpose of quality assurance and active dose reduction. 


\section{CLINICAL RELEVANCE}

- Optimization of the examination technique, e. g. by changing imaging parameters, is often sufficient to reduce radiation exposure enough to satisfy the DRLs while still ensuring diagnostic image quality.

- By implementing suitable optimization steps, the DRLs can be satisfied under consideration of diagnostic image quality even in the case of CT equipment of older generations without iterative image reconstruction.

- Analyzing, reviewing, and comparing the own archived exposure values shows the potential to optimize the applied dose and to use this for the own quality assurance and active dose management.

\section{Conflict of Interest}

The authors declare that they have no conflict of interest.

\section{Literatur}

[1] Internationale Strahlenschutzkommission. Strahlenschutz und Sicherheit in der Medizin; ICRP-Veröffentlichung 73; BfS-SCHR-1999; Wirtschaftsverlag/Verlag für neue Wissenschaft. Bremerhaven, 1999

[2] Europäische Atomgemeinschaft. Richtlinie 97/43/EURATOM des Rates vom 30. Juni 1997 über den Gesundheitsschutz von Personen gegen die Gefahren ionisierender Strahlung bei medizinischer Exposition und zur Aufhebung der Richtlinie 84/466/EURATOM, ABI. L Nr. 180 S. 22.

[3] European Commission. Radiation Protection 109, Guidance on diagnostic reference levels (DRLs) for medical exposures. Luxembourg: Office for Official Publications of the European Communities. 1999

[4] Verordnung über den Schutz vor Schäden durch Röntgenstrahlen (Röntgenverordnung -RöV) vom 8. Januar 1987 (BGBI. I S. 114) in der Fassung der Bekanntmachung vom 30. April 2003 (BGBI. I S 604).

[5] Bundesministerium für Umwelt, Naturschutz, Bau und Reaktorsicherheit. Referat Bundesaufsicht im Strahlenschutz, Qualitätssicherung durch ärztliche und zahnärztliche Stellen, Richtlinie zur Röntgenverordnung und zur Strahlenschutzverordnung, Az. RS II 4 - 11432/6 vom 23. Juni 2015.

[6] Bundesamt für Strahlenschutz. Bekanntmachung der aktualisierten diagnostischen Referenzwerte für diagnostische und interventionelle Röntgenanwendungen, 22. Juni 2016 (BAnz AT 15.07.2016 B8).

[7] Bundesamt für Strahlenschutz. Bekanntmachung der aktualisierten diagnostischen Referenzwerte für diagnostische und interventionelle Röntgenanwendungen, 22. Juni 2010, (BAnz. S. 2594).

[8] Veit R, Guggenberger R, Nosske D et al. Diagnostische Referenzwerte für Röntgenuntersuchungen, Aktualisierung 2010. Radiologe 2010; 50: 907-912
[9] Roller F, Litzlbauer HD, Krombach GA et al. Split-Bolus-Technik in der Computertomografie polytraumatisierter Patienten: Kontrastierung und diagnostische Sicherheit. Fortschr Röntgenstr 2013; 185: VO315_3. doi:10.1055/s-0033-1346440

[10] Kalra MK, Woisetschlager M, Dahlstrom N et al. Radiation dose reduction with Sinogram Affirmed Iterative Reconstruction technique for abdominal computed tomography. Journal of computer assisted tomography 2012; 36: 339-346

[11] Schindera ST, Odedra D, Raza SA et al. Iterative reconstruction algorithm for $\mathrm{CT}$ : can radiation dose be decreased while lowcontrast detectability is preserved? Radiology 2013; 269: 511-518

[12] Bundesärztekammer. Leitlinien der Bundesärztekammer zur Qualitätssicherung in Röntgendiagnostik bzw. Computertomografie. Dtsch Arztebl 2008; 105: A-536/B-480/C-468

[13] WHO. Obesity. Preventing and managing a global epidemic. Report of a WHO Consultation. WHO Technical Report Series. Geneva: WHO. 2000; 894

[14] Bushberg JT. The essential physics of medical imaging. 3rd ed Philadelphia: Wolters Kluwer Health/Lippincott Williams \& Wilkins; 2012: 1030

[15] Mohrs OK, Pettersen SE, Schulze T. High-resolution 3D unenhanced ECG-gated respiratory-navigates MR angiographie of renal arteries: comparison with contrast-enhanced MR angiographie. Am J Roentgenol 2010; 195.6: $1423-1428$

[16] Körner M. Initial clinical results with a new needle screen storage phosphor system in chest radiograms. Fortschr Röntgenstr Stuttgart-New York: () Georg Thieme Verlag KG. 2005. Vol. 177. No. 11

[17] Bulla S, Pache G, Blanke P et al. Strahlendosisreduktion in der Niedrigdosis-CT der Nasennebenhöhlen unter Anwendung der iterativen Bildrekonsturktion: Durchführbarkeit und Bildqualität. Fortschr Röntgenstr 2011; 183 - VO409_1

[18] Reske SU, Braunschweig R, Reske AW et al. Polytrauma-Ganzkörper-CT: Klinisch adaptierter Einsatz unterschiedlich gewichteter CT-Untersuchungsprotokolle. Fortschr Röntgenstr 2018; 190: 1141 - 1151

[19] Geyer LL, Körner M, Harrieder A et al. Dose reduction in 64-row wholebody $\mathrm{CT}$ in multiple trauma: an optimized $\mathrm{CT}$ protocol with iterative image reconstruction on a gemstone-based scintillator. $\mathrm{Br}$ J Radiol 2016; 89: 20160003

[20] Cornier MA, Després JP, Davis N, American Heart Association Obesity Committee of the Council on Nutrition; Physical Activity and Metabolism; Council on Arteriosclerosis; Thrombosis and Vascular Biology; Council on Cardiovascular Disease in the Young; Council on Cardiovascular Radiology and Intervention; Council on Cardiovascular Nursing, Council on Epidemiology and Prevention; Council on the Kidney in Cardiovascular Disease, and Stroke Council. Assessing adiposity et al. A scientific statement from the American Heart Association. Circulation 2011; 124: 1996-2019

[21] Wu Q, Wang Y, Kai H et al. Application of 80-kVp tube voltage, lowconcentration contrast agent and iterative reconstruction in coronary CT angiography: evaluation of image quality and radiation dose. Int J Clin Pract 2016; 70 (Suppl. 9): B50 - B55. doi: 10.1111/ijcp.12852

[22] Zhang J, He B, Wang YL et al. Application of $270 \mathrm{mgl} / \mathrm{mL}$ lodinated Contrast Media in Dual-Source Computed Tomography Coronary Artery Imaging. Acta Cardiol Sin 2014; 30: 538-545 